\title{
Synthesis of $\mathrm{MnMoO}_{4}$ by hydrothermal method and their electrochemical performance
}

\author{
Asiya F. Shaikh, Mohaseen S. Tamboli, Sonali D. Naik, Jalinder D. Ambekar \\ and Bharat B. Kale*
}

Centre for Materials for Electronics Technology, Department of Electronics and Information Technology (DeitY), Govt. of India, Panchawati off Pashan Road, Pune, INDIA

*Email:kbbb1@yahoo.com

As energy storage devices with properties intermediate to those of batteries and electrostatic capacitors, electrochemical supercapacitors exhibit the desirable properties of high power density (ten times more than batteries), fast charging (with seconds), excellent cycling stability, small size and low mass, which make them one of the most promising candidates for next-generation power devices. With characteristics complementary to those of rechargeable batteries and fuel cells, supercapacitors have been used in many applications, such as power back-up, pacemakers, air bags, electrical vehicles, medical, military, aerial lift, public transport buses, etc. Currently, most commercial supercapacitors are made of highsurface-area carbonaceous materials such as graphene, CNT etc. However, these supercapacitors might not provide sufficient energy/power densities or efficiencies, and the specific capacitance severely decreases under high current. Therefore, much attention has been focused on improving the capacitance of supercapacitors, and their performance at high current densities, by constructing complex heterostructures with increased surface areas.

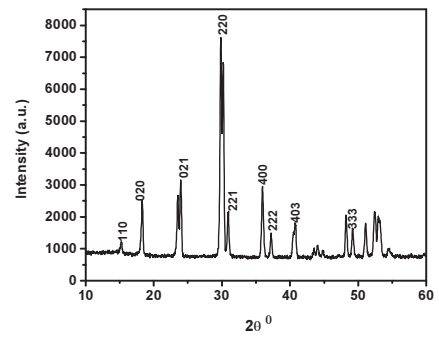

Figure 1: XRD pattern of $\mathrm{MnMoO}_{4}$

A unique and cost effective hydrothermal procedure has been carried out for the synthesis of manganese molybdate $\left(\mathrm{MnMoO}_{4}\right)$ and their electrochemical properties for supercapacitor applications. The microstructure, surface morphology and composition were characterized by using field emission scanning electron microscope (FE-SEM), high resolution-transmission electron microscopy (HR-TEM), X-ray diffraction analysis (XRD) and Raman spectroscopy. The capacitive behavior was investigated by cyclic voltammetry (CV), charge-discharge (CD) cycling and electrochemical impedance spectroscopy (EIS) studies using $2 \mathrm{M} \mathrm{Na}_{2} \mathrm{SO}_{4}$ electrolyte at ambient atmosphere.

XRD pattern and FESEM images of the samples have been shown in Figure 1 and Figure 2, respectively. From the cyclic voltammogram it was identified that the pseudocapacitance arises through the reversible faradic reactions of $\mathrm{Mn}^{3+} \mathrm{Mn}^{2+}$ ionic species. The high performance of the present microstructure can be attributed to the synergetic effect of morphology and chemical composition.

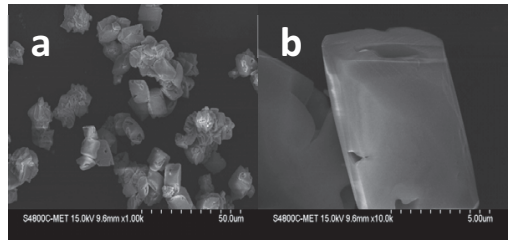

Figure 2: FE-SEM images of (a) as synthesized $\mathrm{MnCo}_{2} \mathrm{O}_{4}$ and (b) $\mathrm{MnxNi1}-\mathrm{xCO}_{2} \mathrm{O}_{4}$

\section{References}

1. L. Li, H. Song, Q. Zhang, J. Yao, X.H. Chen, J. Power Sources 187 (2009) 268.

2. D.S. Patil, J.S. Shaikh, D.S. Dalavi, S.S. Kalagi, P.S. Patil, Mater. Chem. Phys. 128 (2011) 449.

3. S. Biswas, L.T. Drzal, ACS Appl. Mater. Interfaces, 2 (2010) 2293.

4. M. Kumar, K. Singh, S.K. Dhawan, K. Tharanikkarasu, J.S. Chung, B.S. Kong,E.J. Kim, S.H. Hur, Chem. Eng. J. 231 (2013) 397. 